\title{
Coexistence of Extramedullary Plasmacytoma and Fungus Balls in the Paranasal Sinuses: Report a Case
}

\author{
Jhy-Shyan Gau, ${ }^{\mathrm{a}, \mathrm{f}}$, Nan-Han Lu', Lee-Ren Yeha, Tun-Yen Hsu ${ }^{\mathrm{b}}$, \\ Hung-Bo $\mathrm{Wu}^{\mathrm{c}}$, Shyh-An Yeh ${ }^{\mathrm{d}}$, Chung-Ming Kuo
}

\begin{abstract}
Extramedullary plasmacytoma is a rare plasma cell neoplasm that arises most frequently in the head and neck and mainly involves the sinonasal or nasopharyngeal regions. We describe a 68 -year-old patient who developed an extramedullary plasmacytoma coexisting with fungus balls in the paranasal sinuses and was initially treated as fungal sinusitis. This case highlights the importance of surgeons and radiologists being aware of the possibility of concomitant pathology of fungal sinusitis and that appropriate imaging study should be performed before initial treatment.
\end{abstract}

Keywords: Extramedullary plasmacytoma; Fungal sinusitis; Computed tomography; Magnetic Resonance Imaging

\section{Introduction}

Extramedullary plasmacytoma (EMP) is an uncommon plasma cell neoplasm that arises in soft tissues at single or multiple sites [1]. Most EMPs occur in the head and neck region, with the nasosinus, nasopharynx and oropharynx being the most common sites [2]. We report an extremely rare case of

\footnotetext{
Manuscript accepted for publication June 9, 2011

${ }^{a}$ Department of Diagnostic Radiology, E-DA Hospital / I-Shou University, Kaohsiung City, Taiwan, R.O.C.

${ }^{\mathrm{b}}$ Department of Otolaryngology, E-DA Hospital / I-Shou University, Kaohsiung City, Taiwan, R.O.C.

${ }^{\mathrm{c}}$ Department of Hematology-Oncology, E-DA Hospital / I-Shou University, Kaohsiung City, Taiwan, R.O.C.

${ }^{\mathrm{d}}$ Department of Radiation Oncology, E-DA Hospital / I-Shou

University, Kaohsiung City, Taiwan, R.O.C.

${ }^{\mathrm{e}}$ Department of Information Engineering, I-Shou University, Kaohsiung City, Taiwan, R.O.C.

${ }^{f}$ Corresponding author: E-Da Road, Jiau-shu Tsuen, Yan-Chau District,

Kaohsiung City, TAIWAN, 824.

Email: stanfordgau@yahoo.com.tw
}

doi:10.4021/jmc236w
EMP coexisting with fungus balls in the paranasal sinuses that was initially treated as fungal sinusitis.

\section{Case Report}

A 68-year-old diabetic male presented with a history of intermittent epistaxis, especially on the left, for half a year. Frontal headache and nasal stuffiness with nasal pain were complained also. He visited our ENT department for help. Physical examination revealed septal deviation to the left, mucopus over bilateral middle meatuses and left nasal polyposis. Unenhanced sinus computed tomography (CT) scan revealed bilateral paranasal sinusitis with intrasinus calcifications in bilateral maxillary sinuses (Fig. 1a). Bony erosion over the posterior wall of the left maxillary sinus was shown also (Fig. 1b). Functional endoscopic sinus surgery (FESS) was performed under the impression of bilateral fungal sinusitis. However, after removal of the fungus balls, a neoplasm in the left maxillary sinus was found. Biopsies were taken subsequently and the pathological result revealed plasmacytoma. The post-FESS magnetic resonance imaging (MRI) of the paranasal sinuses showed an isointense to slightly hyperintense tumor mass in the left maxillary sinus with extension to the left ethmoid sinus and the left retromaxillary fissure on short inversion time inversion recovery (STIR) images (Fig. 2a). The tumor mass exhibited heterogeneous enhancement after contrast medium administration (Fig. 2b). Finally, the patient was treated with surgical resection and complementary radiotherapy. During the immediate postoperative period, the patient underwent hematologic and oncologic evaluations. Bone marrow biopsy, whole-body skeletal survey and abdominal sonography were normal. The serum $\operatorname{IgG}$ level was $3830 \mathrm{mg} / \mathrm{dl}$, and B2 microglobulin was $0.7 \mathrm{mg} / \mathrm{dl}$. Serum immunoelectrophoresis revealed monocolonal IgG kappa chain. Bence-Jones protein was absent in urine. Thus, a diagnosis of EMP was made.

\section{Discussion}

Plasma cell neoplasms include multiple myloma, solitary 

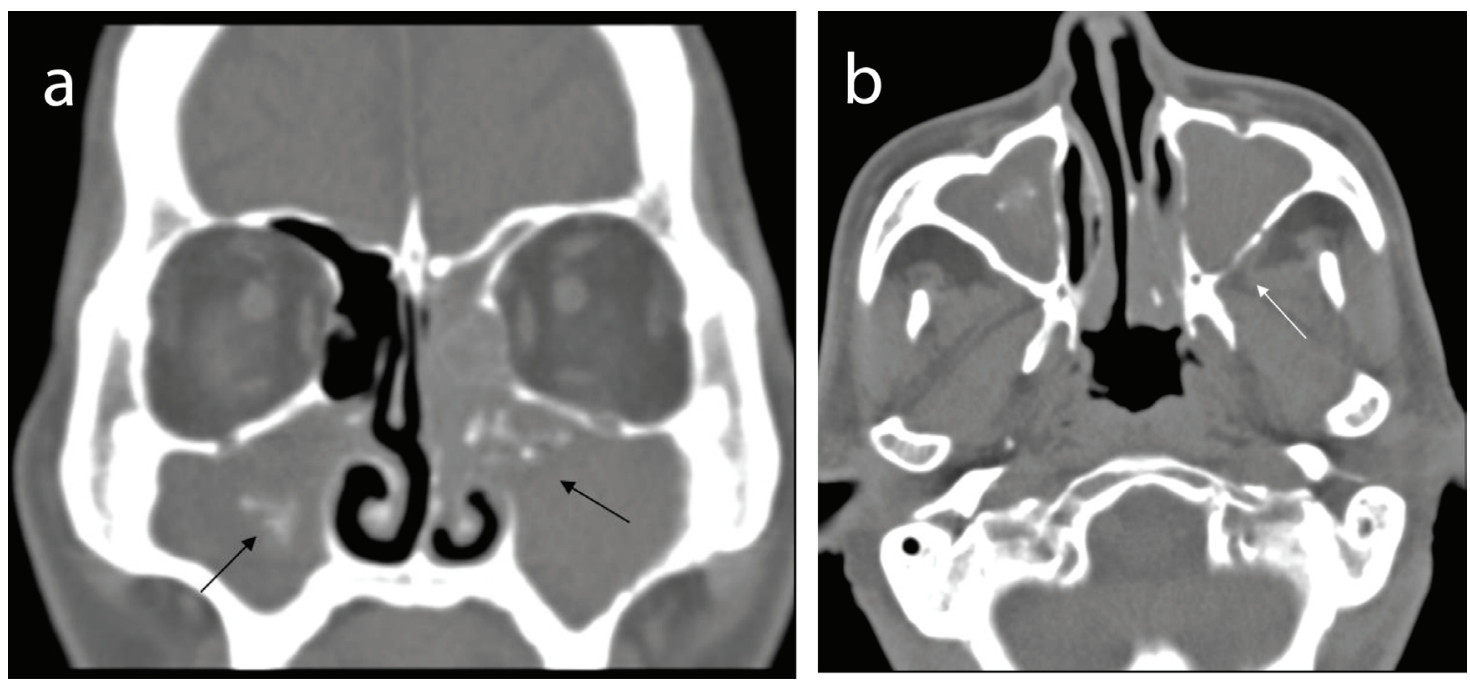

Figure 1. (a) Unrenced CT scan shows opacification of paranasal sinuses with intrasinus calcifications (black arrows) in bilateral maxillary sinuses. (b) Bony erosion (white arrow) over posterior wall of the left maxillary sinus.

plasmacytoma of bone and EMP. EMP accounts for approximately $3 \%$ of all plasma cell malignancies and represents less than $1 \%$ of all head and neck tumors $[3,4]$. The majority of EMP occurs in the head and neck region. Approximately $80 \%$ of EMPs are found in the upper aerodigestic tract (UADT) and their most common sites are the nasosinuses, nasopharynx, oropharynx and larynx. Men are affected 3 to 5 times more than women, and $70 \%$ of EMP patients are between the ages of 50 and 70 . The clinical presentations of these tumors are nonspecific and usually reflect the mass effect of the tumor. The most common findings are soft tissue mass or swelling, airway obstruction, epistaxis, tumor associated local pain and nasal discharge $[1,2,5]$. Our patient presented with epistaxis, frontal headache and nasal pain.

The pathogenesis of EMP is unclear. Wiltshaw suggested that the malignant cells of EMP are of bone marrow
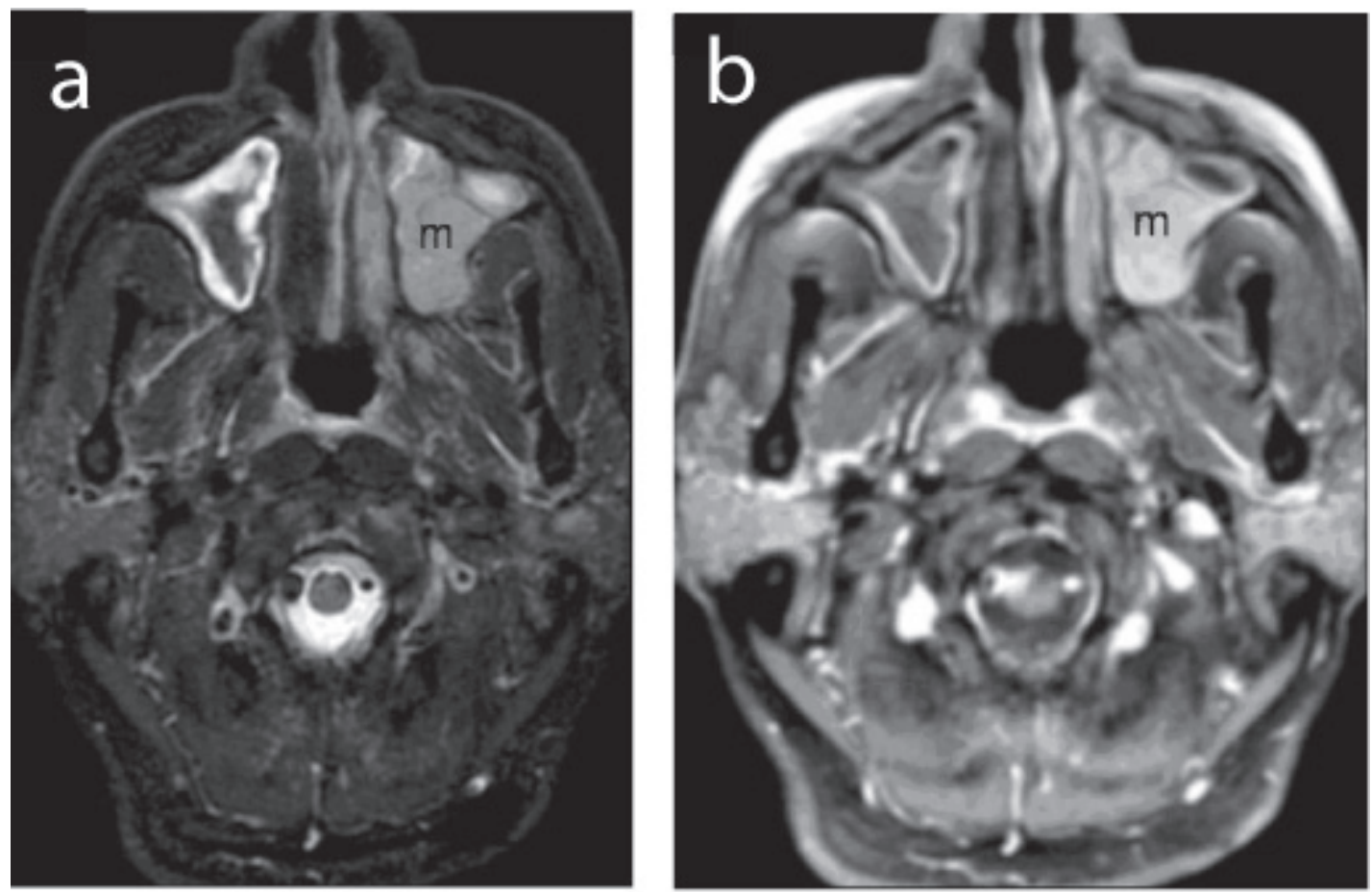

Figure 2. (a) Post-FESS MRI reveals a slightly hyperintense mass $(m)$ in the left maxillary sinus with minimal extra-sinal extension on STIR image. (b) Contrast-enhanced T1-weighted image demonstrating hetrogeneous enhancement of the mass lesion (m). 
origin and have migrated to the peripheral lymphoid tissues and undergone plasmacytoid differentiation. Chronic infection of these tissues may initiate the development of such tumor [1]. Ersoy et al. postulated that chronic stimulation by inhaled irritants or viral infection may be the etiology of soft tissue plasmacytoma [6]. Bush et al. reported three patients with nasal cavity, nasopharyngeal and bilateral tonsillar plasmacytomas who had a history of chronic paranasal sinusitis [7]. Our patient was a case of fungal sinusitis coexistent with EMP. Maybe fungus infection was the chronic stimulation that triggered the neoplastic growth of the EMP. To our knowledge, this is the first case with coexistence of fungus ball and EMP in the paranasal sinus published in the English literature. Tanaka et al. and Shaw et al. reported aspergillosis coexistent with squamous cell carcinoma in the maxillary sinus and sphenoid sinus, respectively $[8,9]$. But fungus ball existing simultaneously with EMP in the paranasal sinus had never been reported. This case emphasizes the importance of treating surgeon to realize the possibility of concomitant pathology of fungal sinusitis.

CT is seen as the "gold standard" imaging modality for sinonasal lesions. CT is superior to delineate the bony anatomy and subtle bony erosion or destruction. MRI offers the advantage of high soft tissue contrast compared to CT [10]. Bony destruction is common for both aspergilus infection and malignant tumor [9]. When CT reveals fungus ball and bony destruction of the paranasal sinus, MRI must be performed to exclude the possibility of coexisting malignancy. MRI not only can demonstrate mucosal thickening of paranasal sinus and centrally located fungus ball but also can differentiate the thickened mucosa from neoplasm. This should be kept in mind by the surgeon during initial treatment of the patient.

The imaging features of EMP are nonspecific, but radiographic assessment is important to evaluate the location, size and extension of the lesion. The EMP may be a bulky soft tissue mass or an infiltrative lesion. It can be locally aggressive and have extensive involvement. CT scan shows soft tissue density with variable enhancement whereas MRI manifests isointensity on T1-weighted images and iso- to hyperintensity on T2-weighted images. With the administration of gadolinium-based contrast medium, moderate to marked enhancement will be displayed $[11,12]$. Imaging alone cannot differentiate EMP from other malignancies of the sinonasal tract such as squamous cell carcinoma, lymphoma, esthesioneuroblastoma, adenocystic carcinoma, rhabdomyosarcoma or metastasis. The diagnosis of EMP requires histological and immunohistochemical evidence. The role of imaging should concentrate on the depiction of local extent of this tumor, presence of lymphadenopathy and early detection of recurrent lesions which will influence clinical management.

The treatment of EMP includes surgery, radiotherapy or a combination of both. The option is aimed at local control and depends on the site, tumor size and extension of the lesion. In 714 cases of EMP in the UADT reviewed by Alexiou et al. [2], surgery or combined therapy (surgery and radiotherapy) had been used as frequently as radiotherapy alone. The median overall survival or recurrence-free survival for patients who had combined therapy (surgery and radiotherapy) was statistically significantly longer than that for those who received surgery or radiotherapy alone. The authors suggested that surgical removal is sufficient if the EMP is present in the soft tissue locally and well operable. When total removal of an EMP is not possible, surgery followed by radiotherapy is recommended [2]. This has been the most common therapeutic strategy for most surgeons in recent years. After treatment of EMP in the UADT, $61.1 \%$ of patients were cured or without systemic involvement. The local recurrence rate was about $22 \%$, and $16.1 \%$ progressed to multiple myeloma [2]. Adjuvent chemotherapy may be used for disseminated disease or conversion to multiple myeloma and in some cases of local recurrence.

\section{Conclusion}

Although the imaging findings are nonspecific, EMP should be included in the differential diagnosis of a paranasal sinus malignant neoplasm. Also, bony erosion can be found in fungal sinusitis, but potential coexistence with a neoplasm must be taken into consideration, and MRI study before initial management is necessary.

\section{References}

1. Wiltshaw E. The natural history of extramedullary plasmacytoma and its relation to solitary myeloma of bone and myelomatosis. Medicine (Baltimore). 1976;55(3):217-238.

2. Alexiou C, Kau RJ, Dietzfelbinger H, Kremer M, Spiess JC, Schratzenstaller B, Arnold W. Extramedullary plasmacytoma: tumor occurrence and therapeutic concepts. Cancer. 1999;85(11):2305-2314.

3. Knowling MA, Harwood AR, Bergsagel DE. Comparison of extramedullary plasmacytomas with solitary and multiple plasma cell tumors of bone. J Clin Oncol. 1983;1(4):255-262.

4. Fu YS, Perzin KH. Nonepithelial tumors of the nasal cavity, paranasal sinuses and nasopharynx. A clinicopathologic study. IX. Plasmacytomas. Cancer. 1978;42(5):2399-2406.

5. Kapadia SB, Desai U, Cheng VS. Extramedullary plasmacytoma of the head and neck. A clinicopathologic study of 20 cases. Medicine (Baltimore). 1982;61(5):317-329.

6. Ersoy O, Sanlier T, Yigit O, Halefoglu AM, Ucak S, Altuntas Y. Extramedullary plasmacytoma of the maxillary sinus. Acta Otolaryngol. 2004;124(5):642-644. 
7. Bush SE, Goffinet DR, Bagshaw MA. Extramedullary plasmacytoma of the head and neck. Radiology. 1981;140(3):801-805.

8. Tanaka T, Nishioka K, Naito M, Masuda Y, Ogura Y. Coexistence of aspergillosis and squamous-cell carcinoma in the maxillary sinus proven by preoperative cytology. Acta Cytol. 1985;29(1):73-78.

9. Shaw CL, McCleave M, Wormald PJ. Unusual presentations of isolated sphenoid fungal sinusitis. J Laryngol Otol. 2000;114(5):385-388.
10. Eggesbo HB. Radiological imaging of inflammatory lesions in the nasal cavity and paranasal sinuses. Eur Radiol. 2006;16(4):872-888.

11. Vogl TJ, Steger W, Grevers G, Balzer J, Mack M, Felix R. MR characteristics of primary extramedullary plasmacytoma in the head and neck. AJNR Am J Neuroradiol. 1996;17(7):1349-1354.

12. Ching AS, Khoo JB, Chong VF. CT and MR imaging of solitary extramedullary plasmacytoma of the nasal tract. AJNR Am J Neuroradiol. 2002;23(10):1632-1636. 